\title{
Antiperiodic Problems for Nonautonomous Parabolic Evolution Equations
}

\author{
R. N. Wang' and Y. Zhou' \\ ${ }^{1}$ Department of Applied Mathematics, Guangdong University of Foreign Studies, Guangzhou 510420, China \\ ${ }^{2}$ Department of Mathematics, Xiangtan University, Xiangtan, Hunan 411105, China \\ Correspondence should be addressed to Y. Zhou; yzhou@xtu.edu.cn
}

Received 10 April 2014; Accepted 4 May 2014; Published 22 May 2014

Academic Editor: Hui-Sheng Ding

Copyright (c) 2014 R. N. Wang and Y. Zhou. This is an open access article distributed under the Creative Commons Attribution License, which permits unrestricted use, distribution, and reproduction in any medium, provided the original work is properly cited.

This work focuses on the antiperiodic problem of nonautonomous semilinear parabolic evolution equation in the form $u \prime(t)=$ $A(t) u(t)+f(t, u(t)), t \in \mathbb{R}, u(t+T)=-u(t), t \in \mathbb{R}$, where $(A(t))_{t \in \mathbb{R}}$ (possibly unbounded), depending on time, is a family of closed and densely defined linear operators on a Banach space $X$. Upon making some suitable assumptions such as the Acquistapace and Terreni conditions and exponential dichotomy on $(A(t))_{t \in \mathbb{R}}$, we obtain the existence results of antiperiodic mild solutions to such problem. The antiperiodic problem of nonautonomous semilinear parabolic evolution equation of neutral type is also considered. As sample of application, these results are applied to, at the end of the paper, an antiperiodic problem for partial differential equation, whose operators in the linear part generate an evolution family of exponential stability.

\section{Introduction and Motivation}

Antiperiodic problems have recently proved to be valuable tools in the modelling of many phenomena in physical processes. For the background on this class of problems we refer the reader to [1-3] and the references therein. For this reason, much attention is attracted by questions of existence of antiperiodic solutions to the various antiperiodic problems represented by linear and nonlinear abstract evolution equations since the work of Okochi [4] in 1988 (see also [5, 6]). The literature related to such problems is quite extensive; see, for instance, Haraux [7] for nonlinear first order evolution equations in Hilbert spaces and Aftabizadeh et al. [8] and Aizicovici and Pavel [9] for second order evolution equations in Hilbert and Banach spaces. In particular, using the maximal monotone property of the derivative operator with antiperiodic conditions and the theory of pseudomonotone perturbations of maximal monotone mappings, Liu [10] recently studied the antiperiodic problem for nonlinear evolution equation with nonmonotone perturbation of the form

$$
\begin{aligned}
u^{\prime}(t)+A u(t)+G u(t) & =f(t), \quad \text { a.e. } t \in(0, T), \\
u(T) & =-u(0)
\end{aligned}
$$

in a real reflexive Banach space $V$, where $A$ is monotone and $G$ is not. For more details about development and applications along this line, see, for example, $[1,11-13]$ and the references therein. Let us note that equations in the research mentioned above are all autonomous.

Motivated by these works, in this paper we will carry out our investigation to the semilinear nonautonomous parabolic evolution equation having the form

$$
u^{\prime}(t)=A(t) u(t)+f(t, u(t)), \quad t \in \mathbb{R},
$$

subject to antiperiodic condition

$$
u(t+T)=-u(t), \quad t \in \mathbb{R}
$$

in the Banach space $X$. Here, $\mathbb{R}$ stands for the set of real numbers, $(A(t))_{t \in \mathbb{R}}$ (possibly unbounded), depending on time, is a family of closed and densely defined linear operators on $X$ and has domains $(D(A(t)))_{t \in \mathbb{R}}$, and $f$ is a given function to be specified later.

Note also that the problem (2)-(3) has been considered by Wang [14] under different situations, in which the author proved the existence of antiperiodic mild (strict) solutions in 
the case of (2) being a mild (strict) dissipative differential equation by using the modular degree theorem. The line, which we will go along in this study, is that we establish some results concerning the existence of antiperiodic mild solutions to the problem (2)-(3) under new criteria (without the assumption of (2) being dissipative). Then, we also consider the antiperiodic mild solutions to nonautonomous semilinear parabolic evolution equation of neutral type

$$
\frac{d}{\mathrm{~d} t}[u(t)-F(t, u(t))]=A(t) u(t)+f(t, u(t)), \quad t \in \mathbb{R} .
$$

Finally, as a sample of possible applications, we give a result on the existence of antiperiodic mild solutions to a partial differential equation with homogeneous Dirichlet boundary condition, whose operator in the linear part generates an evolution family of exponential stability. As can be seen, our results extend and unify many existing results in this area. Banach's contraction principle, Schauder's fixed point theorem, and Krasnoselskii's fixed point theorem, as well as the theory of evolution families such as exponential dichotomy techniques, are employed in our approach. Note also that the conditions we used in this paper are quite different from those in [14].

We organize this paper as follows. We present some definitions and preliminary facts in Section 2. In Section 3, starting with introducing the assumptions that are needed in the proofs of our main results, we then establish some existence theorems of antiperiodic mild solutions to the problem (2)-(3). In Section 4, we extend the result obtained in Section 3 to the neutral problem (4). An example is given to illustrate the theorem in Section 5.

\section{Preliminaries}

Throughout this paper, $X$ is assumed to be a Banach space with norm $\|\cdot\|$ and $\mathscr{L}(X)$ stands for the Banach space of all bounded linear operators from $X$ to $X$ equipped with its natural topology. Denote by $C_{b}(\mathbb{R} ; X)$ the Banach space of all bounded, continuous functions from $\mathbb{R}$ to $X$ equipped with the sup norm

$$
\|u\|_{C_{b}(\mathbb{R} ; X)}=\sup \{\|u(t)\| ; t \in \mathbb{R}\}
$$

by $L(0, T ; X)$ the Banach space of all Bocher integrable functions from $[0, T]$ to $X$ equipped with the norm

$$
\|u\|_{L(0, T ; X)}=\int_{0}^{T}\|u(t)\| \mathrm{d} t
$$

and by $L_{\text {loc }}(\mathbb{R} ; X)$ the set of all locally Bocher integrable functions from $\mathbb{R}$ to $X$.

A function $u \in C_{b}(\mathbb{R} ; X)$ is said to be $T$-antiperiodic if

$$
u(t+T)=-u(t), \quad \forall t \in \mathbb{R}
$$

By $P_{T A}(\mathbb{R} ; X)$, we denote the set of all $T$-antiperiodic functions from $\mathbb{R}$ to $X$. It is easy to see that $P_{T A}(\mathbb{R} ; X)$, equipped with the sup norm, is a Banach space. For every $r>0$, write

$$
\Omega_{r}=\left\{u \in P_{T A}(\mathbb{R}, X) ;\|u\|_{P_{T A}(\mathbb{R} ; X)} \leq r\right\},
$$

which is convex closed subset of $P_{T A}(\mathbb{R}, X)$.

The following lemma provides some useful information on compactness criterion, which can be regarded as an extension of the known Arzéla-Ascoli theorem.

Lemma 1 (see [15, Lemma 3.1]). A set $D \subseteq P_{T A}(\mathbb{R} ; X)$ is relatively compact in $P_{T A}(\mathbb{R} ; X)$ if $D$ is equicontinuous and the set $D(t):=\{u(t) ; u \in D\}$ is relatively compact in $X$ for every $t \in \mathbb{R}$.

Definition 2. A family of bounded linear operators $U=$ $\{U(t, s)\}_{t \geq s}$ on a Banach space $X$ is called a strongly continuous evolution family if

(1) $U(t, r) U(r, s)=U(t, s)$ and $U(t, t)=I$ for all $t \geq r \geq s$ and $t, r, s \in \mathbb{R}$,

(2) the map $(t, s) \mapsto U(t, s) x$ is continuous for all $x \in X$, $t \geq s$ and $t, s \in \mathbb{R}$.

Throughout the paper, we assume that $(A(t))_{t \in \mathbb{R}}$ is a family of closed and densely defined operators on $X$, which satisfies the conditions of Acquistapace and Terreni $\left(\mathrm{AT}_{1}\right)$ and $\left(\mathrm{AT}_{2}\right)$.

$\left(\mathrm{AT}_{1}\right) A(t)$ are linear operators on $X$ and there are constants $\lambda_{0} \geq 0, \theta \in((\pi / 2), \pi)$, and $K_{1} \geq 0$ such that $\Sigma_{\theta} \cup\{0\} \subset$ $\rho\left(A(t)-\lambda_{0}\right)$ and for all $\lambda \in \Sigma_{\theta} \cup\{0\}$ and $t \in \mathbb{R}$,

$$
\left\|R\left(\lambda, A(t)-\lambda_{0}\right)\right\|_{\mathscr{L}(X)} \leq \frac{K_{1}}{1+|\lambda|} .
$$

$\left(\mathrm{AT}_{2}\right)$ There are constants $K_{2} \geq 0$ and $\alpha, \beta \in(0,1]$ with $\alpha+\beta>1$ such that for all $\lambda \in \Sigma_{\theta}$ and $t, s \in \mathbb{R}$

$$
\begin{aligned}
\|(A(t) & \left.-\lambda_{0}\right) R\left(\lambda, A(t)-\lambda_{0}\right) \\
& \times\left[R\left(\lambda_{0}, A(t)\right)-R\left(\lambda_{0}, A(s)\right)\right] \|_{\mathscr{L}(X)} \\
\leq & \frac{K_{2}|t-s|^{\alpha}}{|\lambda|^{\beta}} .
\end{aligned}
$$

Here $\Sigma_{\theta}:=\{\lambda \in \mathbb{C} \backslash\{0\} ;|\lambda| \leq \theta\}$.

Conditions $\left(\mathrm{AT}_{1}\right)$ and $\left(\mathrm{AT}_{2}\right)$, which are initiated by Acquistapace and Terreni $[16,17]$ for $\lambda_{0}=0$, are well understood and widely used in the literature.

Remark 3. It should be mentioned that when $(A(t))_{t \in \mathbb{R}}$ has a constant domain $D(A(t)),\left(\mathrm{AT}_{2}\right)$ can be replaced with the following: there exist constants $K_{2}>0,0<\mu \leq 1$ such that

$$
\left\|(A(t)-A(s)) R\left(\lambda_{0}, A(r)\right)\right\|_{\mathscr{L}(X)} \leq K_{2}|t-s|^{\mu}
$$

for all $s, t, r \in \mathbb{R}$ (see, e.g., $[18,19])$.

By an obvious rescaling from [16, Theorem 2.3] and [20, Theorem 2.1] (see also [17, 21]), it follows that conditions 
$\left(\mathrm{AT}_{1}\right)$ and $\left(\mathrm{AT}_{2}\right)$ ensure that there exists a unique evolution family $\{U(t, s)\}_{t \geq s}$ on $X$ such that

(I) $U(\cdot, s) \in C^{1}((s, \infty), \mathscr{L}(X)), \partial U(t, s) / \partial t=A(t) U(t, s)$ for $t>s$, and

$$
\left\|A(t)^{k} U(t, s)\right\|_{\mathscr{L}(X)} \leq C(t-s)^{-k}
$$

for $0<t-s \leq 1, k=0,1$;

(II) $\partial^{+} U(t, s) x / \partial s=-U(t, s) A(s) x$ for $t>s$ and $x \in$ $D(A(s))$ with $A(s) x \in \overline{D(A(s))}$.

In this case we say that $(A(t))_{t \in \mathbb{R}}$ generates the evolution family $U$.

Definition 4. An evolution family $U=\{U(t, s)\}_{t \geq s}$ is called hyperbolic (or has exponential dichotomy) if there are projections $\mathscr{P}(t), t \in \mathbb{R}$, that are uniformly bounded and strongly continuous in $t$, and constants $N, \delta>0$ such that

(a) $U(t, s) \mathscr{P}(s)=\mathscr{P}(t) U(t, s)$ for all $t \geq s$;

(b) the restriction $U_{\mathbb{Q}}(t, s): \mathbb{Q}(s) X \quad \rightarrow \quad \mathbb{Q}(t) X$ is invertible for all $t \geq s$ (and we set $U_{Q}(s, t)=$ $\left.U_{Q}(t, s)^{-1}\right)$;

(c) $\|U(t, s) \mathscr{P}(s)\|_{\mathscr{L}(X)} \leq N e^{-\delta(t-s)}$ and $\left\|U_{\mathscr{Q}}(s, t) \mathscr{Q}(t)\right\|_{\mathscr{L}(X)} \leq$ $N e^{-\delta(t-s)}$ for all $t \geq s$.

Here and below $\mathbb{Q}=I-\mathscr{P}$. Specially, if $\mathscr{P}(t)=I$ for $t \in \mathbb{R}$, then $U$ is said to be exponentially stable.

Exponential dichotomy is a classical concept in the study of the long-term behavior of evolution equations; see [22-24] and references therein.

\section{The Existence of Antiperiodic Mild Solutions}

In this section we establish some existence theorems of antiperiodic mild solutions to the problem (2)-(3).

To prove our main results, we introduce the following assumptions. For sake of brevity, put $B_{r}:=\{x \in X ;\|x\| \leq r\}$ for some $r>0$.

$\left(\mathrm{H}_{1}\right)$ The evolution family $U=\{U(t, s)\}_{t \geq s}$, generated by $(A(t))_{t \in \mathbb{R}}$, is hyperbolic. Moreover, $U(t+T, s+T) \mathscr{P}(s+$ $T)=U(t, s) \mathscr{P}(s)$ for all $t \geq s$, and $U_{\mathscr{Q}}(t+T, s+T) Q(s+$ $T)=U_{\mathscr{Q}}(t, s) \mathscr{Q}(s)$ for all $t \leq s$.

$\left(\mathrm{H}_{2}\right)$ The function $f: \mathbb{R} \times X \rightarrow X$ satisfies the following conditions.

(i) $f(\cdot, u)$ is measurable for each $u \in X$ and $f(t+$ $T,-u)=-f(t, u)$ for all $t \in \mathbb{R}, u \in X$.

(ii) There exists a constant $L_{f}>0$ with $2 N L_{f}<\delta$ such that

$$
\|f(t, u)-f(t, v)\| \leq L_{f}\|u-v\|
$$

for a.e. $t \in[0, T]$ and all $u, v \in X$.
$\left(\mathrm{H}_{3}\right) \quad$ (i) The function $f: \mathbb{R} \times X \rightarrow X$ is a Carathéodory function; that is, for every $u \in X, f(\cdot, u)$ is measurable and for a.e. $t \in \mathbb{R}, f(t, \cdot)$ is continuous, and $f(t+T,-u)=-f(t, u)$ for all $t \in \mathbb{R}, u \in X$.

(ii) There exists a function $\Phi_{r}(\cdot) \in L\left(0, T ; \mathbb{R}^{+}\right)$such that

$$
\|f(t, u)\| \leq \Phi_{r}(t)
$$

for a.e. $t \in[0, T]$ and all $u \in B_{r}$, and

$$
\liminf _{r \rightarrow+\infty} \frac{\int_{0}^{T} \Phi_{r}(t) \mathrm{d} t}{r}=\sigma_{1}
$$

$$
\text { with } N \sigma_{1}<1-e^{-\delta T} \text {. }
$$

$\left(\mathrm{H}_{4}\right) U(t, s)$ is compact for $t>s$.

Remark 5. From $\left(\mathrm{H}_{1}\right)$ it is clear that $U(t+T, s+T)=U(t, s)$ for all $t \geq s$.

Definition 6. A mild solution to (2) is a function $u \in C_{b}(\mathbb{R} ; X)$ satisfying the integral equation

$$
u(t)=U(t, s) u(s)+\int_{s}^{t} U(t, \tau) f(\tau, u(\tau)) \mathrm{d} \tau
$$

for all $t>s$ and $s \in \mathbb{R}$.

Before stating the existence theorem, we first prove the following lemma.

Lemma 7. Let the assumption $\left(H_{1}\right)$ be satisfied. Given $g \in$ $L_{\text {loc }}(\mathbb{R} ; X)$, suppose that $g(s+T)=-g(s)$ for a.e. $s \in \mathbb{R}$. Define

$$
\begin{aligned}
(\Phi g)(t):= & \int_{-\infty}^{t} U(t, \tau) \mathscr{P}(\tau) g(\tau) \mathrm{d} \tau \\
& -\int_{t}^{+\infty} U_{\mathscr{Q}}(t, \tau) \mathscr{Q}(\tau) g(\tau) \mathrm{d} \tau, \quad t \in \mathbb{R} .
\end{aligned}
$$

Then $(\Phi g)(t)$ is well defined for each $t \in \mathbb{R}$ and $\Phi g$ belongs to $P_{T A}(\mathbb{R} ; X)$.

Proof. From (c) and our assumptions on $g$ it follows that for each $t \in \mathbb{R}$,

$$
\begin{aligned}
\|(\Phi g)(t)\| \leq & \int_{-\infty}^{t}\|U(t, \tau) \mathscr{P}(\tau) g(\tau)\| \mathrm{d} \tau \\
& +\int_{t}^{+\infty}\left\|U_{Q}(t, \tau) \mathcal{Q}(\tau) g(\tau)\right\| \mathrm{d} \tau \\
\leq & N \int_{-\infty}^{t} e^{-\delta(t-\tau)}\|g(\tau)\| \mathrm{d} \tau \\
& +N \int_{t}^{+\infty} e^{\delta(t-\tau)}\|g(\tau)\| \mathrm{d} \tau
\end{aligned}
$$




$$
\begin{aligned}
& \leq \frac{N}{1-e^{-\delta T}}\left(\int_{t-T}^{t}\|g(\tau)\| \mathrm{d} \tau+\int_{t}^{t+T}\|g(\tau)\| \mathrm{d} \tau\right) \\
& =\frac{2 N}{1-e^{-\delta T}}\|g\|_{L((0, T) ; X)}
\end{aligned}
$$

which implies that $(\Phi g)(t)$ is well defined for $t \in \mathbb{R}$ and $\Phi g$ is bounded.

To prove $\Phi g$ belongs to $P_{T A}(\mathbb{R} ; X)$, we first verify the continuity of $\Phi g$. For fixed $t_{0} \in \mathbb{R}$ and $h \in \mathbb{R}$, we obtain upon changing of variables that

$$
\begin{aligned}
& (\Phi g)\left(t_{0}+h\right)-(\Phi g)\left(t_{0}\right) \\
& =\int_{-\infty}^{t_{0}} U\left(t_{0}+h, \tau+h\right) \mathscr{P}(\tau+h) g(\tau+h) \mathrm{d} \tau \\
& \quad-\int_{t_{0}}^{+\infty} U_{\mathscr{Q}}\left(t_{0}+h, \tau+h\right) \mathcal{Q}(\tau+h) g(\tau+h) \mathrm{d} \tau \\
& \quad-\int_{-\infty}^{t_{0}} U\left(t_{0}, \tau\right) \mathscr{P}(\tau) g(\tau) \mathrm{d} \tau \\
& \quad+\int_{t_{0}}^{+\infty} U_{\mathscr{Q}}\left(t_{0}, \tau\right) \mathscr{Q}(\tau) g(\tau) \mathrm{d} \tau .
\end{aligned}
$$

Thus, we have

$$
\begin{aligned}
& \left\|(\Phi g)\left(t_{0}+h\right)-(\Phi g)\left(t_{0}\right)\right\| \\
& \leq \int_{-\infty}^{t_{0}}\left\|U\left(t_{0}+h, \tau+h\right) \mathscr{P}(\tau+h)(g(\tau+h)-g(\tau))\right\| \mathrm{d} \tau \\
& \quad+\int_{t_{0}}^{+\infty} \| U_{\mathscr{Q}}\left(t_{0}+h, \tau+h\right) \mathscr{Q}(\tau+h) \\
& \quad \times(g(\tau+h)-g(\tau)) \| \mathrm{d} \tau \\
& \quad+\int_{-\infty}^{t_{0}} \|\left(U\left(t_{0}+h, \tau+h\right) \mathscr{P}(\tau+h)-U(t, \tau) \mathscr{P}(\tau)\right) \\
& \quad \times g(\tau) \| \mathrm{d} \tau \\
& +\int_{t_{0}}^{+\infty} \|\left(U_{\mathscr{Q}}\left(t_{0}+h, \tau+h\right) \mathscr{Q}(\tau+h)-U\left(t_{0}, \tau\right) \mathscr{Q}(\tau)\right) \\
& \quad \times g(\tau) \| \mathrm{d} \tau \\
& :=I_{1}+I_{2}+I_{3}+I_{4} .
\end{aligned}
$$

Noticing that $\left\|U\left(t_{0}+h, \tau+h\right) \mathscr{P}(\tau+h)\right\| \leq N e^{-\delta\left(t_{0}-\tau\right)}$, we have

$$
I_{1} \leq \frac{N}{1-e^{-\delta T}} \int_{0}^{T}\|g(s+h)-g(s)\| \mathrm{d} s,
$$

which yields $\lim _{h \rightarrow 0} I_{1}=0$. An analogue argument shows $\lim _{h \rightarrow 0} I_{2}=0$.

Since

$$
\{(t, \tau):-\infty<\tau \leq t<+\infty\} \ni(t, \tau) \longrightarrow U(t, \tau) \mathscr{P}(\tau)
$$

is strongly continuous, we obtain

$$
\lim _{h \rightarrow 0} U\left(t_{0}+h, \tau+h\right) \mathscr{P}(\tau+h) g(\tau)=U\left(t_{0}, \tau\right) \mathscr{P}(\tau) g(\tau)
$$

for all $\tau \leq t_{0}, \tau \in \mathbb{R}$. This, together with (c), yields that

$$
\begin{aligned}
& \left\|\left(U\left(t_{0}+h, \tau+h\right) \mathscr{P}(\tau+h)-U\left(t_{0}, \tau\right) \mathscr{P}(\tau)\right) g(\tau)\right\| \\
& \quad \leq 2 N e^{-\delta\left(t_{0}-\tau\right)}\|g\|_{C_{b}(\mathbb{R} ; X)}
\end{aligned}
$$

for all $\tau \leq t_{0}, \tau \in \mathbb{R}$. Now, using the Lebesgue dominated convergence theorem we obtain $\lim _{h \rightarrow 0} I_{3}=0$. Similarly, we can show that $\lim _{h \rightarrow 0} I_{4}=0$.

Next, it remains to prove that $\Phi g$ is $T$-antiperiodic. Noting that $g(\tau+T)=-g(\tau)$ for a.e. $\tau \in \mathbb{R}$, we have

$$
\begin{aligned}
(\Phi g)(t+T)= & \int_{-\infty}^{t+T} U(t+T, \tau) \mathscr{P}(\tau) g(\tau) \mathrm{d} \tau \\
& -\int_{t+T}^{+\infty} U_{\mathscr{Q}}(t+T, \tau) \mathscr{Q}(\tau) g(\tau) \mathrm{d} \tau \\
= & -\int_{-\infty}^{t} U(t, \tau) \mathscr{P}(\tau) g(\tau) \mathrm{d} \tau \\
& +\int_{t}^{+\infty} U_{\mathscr{Q}}(t, \tau) \mathscr{Q}(\tau) g(\tau) \mathrm{d} \tau \\
= & -(\Phi g)(t)
\end{aligned}
$$

for any $t \in \mathbb{R}$. Therefore, we can conclude that $\Phi g$ belongs to $P_{T A}(\mathbb{R} ; X)$. This completes the proof.

Now we are ready to state the first main result.

Theorem 8. Let $\left(H_{1}\right)$ and $\left(H_{2}\right)$ hold. Then the problem (2)-(3) has a unique $T$-antiperiodic mild solution.

Proof. Set, for $u \in P_{T A}(\mathbb{R} ; X), g(\cdot)=f(\cdot, u(\cdot))$. It easily follows from $\left(\mathrm{H}_{2}\right)$ that the function $g$ satisfies the conditions of Lemma 7. From this, we obtain that the mapping $\Gamma$, defined by

$$
\begin{array}{r}
(\Gamma u)(t)=\int_{-\infty}^{t} U(t, \tau) \mathscr{P}(\tau) f(\tau, u(\tau)) \mathrm{d} \tau \\
-\int_{t}^{+\infty} U_{\mathscr{Q}}(t, \tau) \mathbb{Q}(\tau) f(\tau, u(\tau)) \mathrm{d} \tau, \\
t \in \mathbb{R}, \quad u \in P_{T A}(\mathbb{R} ; X),
\end{array}
$$

is well defined and maps $P_{T A}(\mathbb{R} ; X)$ into itself. 
To prove the theorem, we first show that $\Gamma$ has a unique fixed point in $P_{T A}(\mathbb{R}, X)$. Let $u, v \in P_{T A}(\mathbb{R} ; X)$. Then, by $\left(\mathrm{H}_{2}\right)$ we have

$$
\begin{aligned}
& \|(\Gamma u)(t)-(\Gamma v)(t)\| \\
& \quad \leq \int_{-\infty}^{t}\|U(t, \tau) \mathscr{P}(\tau)(f(\tau, y(\tau))-f(\tau, v(\tau)))\| \mathrm{d} \tau \\
& \quad+\int_{t}^{+\infty}\left\|U_{\mathscr{Q}}(t, \tau) \mathscr{Q}(\tau)(f(\tau, u(\tau))-f(\tau, v(\tau)))\right\| \mathrm{d} \tau \\
& \quad \leq \frac{2 N L_{f}}{\delta}\|u-v\|_{P_{T A}(\mathbb{R} ; X)} .
\end{aligned}
$$

Consequently,

$$
\|\Gamma u-\Gamma v\|_{P_{T A}(\mathbb{R} ; X)} \leq \frac{2 N L_{f}}{\delta}\|u-v\|_{P_{T A}(\mathbb{R} ; X)},
$$

which, together with our assumption $2 N L_{f}<\delta$, implies that $\Gamma$ is a strict contraction on $P_{T A}(\mathbb{R} ; X)$. Thus, using the Banach contraction principle we conclude that $\Gamma$ has a unique fixed point in $P_{T A}(\mathbb{R}, X)$.

To the end of the proof, we will prove that $u \in P_{T A}(\mathbb{R}, X)$ is a mild solution of (2) if and only if it is a fixed point of $\Gamma$.

We first suppose that $u \in P_{T A}(\mathbb{R} ; X)$ is a mild solution of (2); that is, $u$ satisfies the integral equation

$$
u(t)=U(t, s) u(s)+\int_{s}^{t} U(t, \tau) f(\tau, u(\tau)) \mathrm{d} \tau
$$

for all $t>s$ and $s \in \mathbb{R}$. From this and (c), it immediately follows that

$$
\begin{aligned}
& \mathscr{P}(t) u(t)=\int_{-\infty}^{t} U(t, \tau) \mathscr{P}(\tau) f(\tau, u(\tau)) \mathrm{d} \tau, \quad t \in \mathbb{R}, \\
& \mathcal{Q}(t) u(t)=\int_{+\infty}^{t} U_{\mathscr{Q}}(t, \tau) \mathscr{Q}(\tau) f(\tau, u(\tau)) \mathrm{d} \tau, \quad t \in \mathbb{R} .
\end{aligned}
$$

So, one has

$$
\begin{aligned}
u(t)= & (\mathscr{P}(t)+\mathscr{Q}(t)) u(t) \\
= & \int_{-\infty}^{t} U(t, \tau) \mathscr{P}(\tau) f(\tau, u(\tau)) \mathrm{d} \tau \\
& -\int_{t}^{+\infty} U_{\mathscr{Q}}(t, \tau) \mathscr{Q}(\tau) f(\tau, u(\tau)) \mathrm{d} \tau, \quad t \in \mathbb{R} .
\end{aligned}
$$

This proves that $u$ is a fixed point of $\Gamma$.

Conversely, if $u \in P_{T A}(\mathbb{R} ; X)$ is a fixed point of $\Gamma$, then $u$ satisfies the integral equations

$$
\begin{aligned}
u(s)= & \int_{-\infty}^{s} U(s, \tau) \mathscr{P}(\tau) f(\tau, u(\tau)) \mathrm{d} \tau \\
& -\int_{s}^{+\infty} U_{Q}(s, \tau) \mathcal{Q}(\tau) f(\tau, u(\tau)) \mathrm{d} \tau, \quad s \in \mathbb{R} .
\end{aligned}
$$

For any $t>s, s \in \mathbb{R}$, we obtain upon multiplying both sides of $(32)$ by $U(t, s)$ that

$$
\begin{aligned}
U(t, s) u(s)= & \int_{-\infty}^{s} U(t, \tau) \mathscr{P}(\tau) f(\tau, u(\tau)) \mathrm{d} \tau \\
& -\int_{s}^{+\infty} U_{Q}(t, \tau) \mathcal{Q}(\tau) f(\tau, u(\tau)) \mathrm{d} \tau \\
= & \int_{-\infty}^{t} U(t, \tau) \mathscr{P}(\tau) f(\tau, u(\tau)) \mathrm{d} \tau \\
& -\int_{s}^{t} U(t, \tau) \mathscr{P}(\tau) f(\tau, u(\tau)) \mathrm{d} \tau \\
& -\int_{t}^{+\infty} U_{\mathscr{Q}}(t, \tau) \mathbb{Q}(\tau) f(\tau, u(\tau)) \mathrm{d} \tau \\
& -\int_{s}^{t} U_{\mathscr{Q}}(t, \tau) \mathscr{Q}(\tau) f(\tau, u(\tau)) \mathrm{d} \tau \\
= & u(t)-\int_{s}^{t} U(t, \tau) f(\tau, u(\tau)) \mathrm{d} \tau,
\end{aligned}
$$

which implies that $u$ is a mild solution to problem (2).

Now, according to the discussed above we deduce that the problem (2)-(3) has a unique $T$-antiperiodic mild solution. The proof is completed.

Now we are in a position to prove our second existence result of antiperiodic mild solutions for the problem (2)-(3).

Theorem 9. Let $\left(H_{1}\right),\left(H_{3}\right)$, and $\left(H_{4}\right)$ hold with $\mathscr{P}(t)=I$ for $t \in \mathbb{R}$. Then the problem (2)-(3) has at least one $T$-antiperiodic mild solution.

Proof. Let us define the mapping $\Gamma$ by

$$
\begin{array}{r}
(\Gamma u)(t)=\int_{-\infty}^{t} U(t, \tau) f(\tau, u(\tau)) \mathrm{d} \tau, \quad t \in \mathbb{R}, \\
u \in P_{T A}(\mathbb{R} ; X) .
\end{array}
$$

We first notice, thanks to assumptions $\left(\mathrm{H}_{1}\right)$ and $\left(\mathrm{H}_{3}\right)(\mathrm{i})$ and Lemma 7, that $\Gamma$ is well defined and maps $P_{T A}(\mathbb{R} ; X)$ into itself.

Next, by applying Schauder's fixed point theorem we show that $\Gamma$ has at least one fixed point in $P_{T A}(\mathbb{R}, X)$. From $\left(\mathrm{H}_{3}\right)$ (ii) it is easy to see that there exists some $k_{0}>0$ such that

$$
\frac{N}{1-e^{-\delta T}} \int_{0}^{T} \Phi_{k_{0}}(\tau) \mathrm{d} \tau \leq k_{0} .
$$


Using this, a direct calculation yields that, for every $u \in \Omega_{k_{0}}$ and all $t \in \mathbb{R}$,

$$
\begin{aligned}
\|(\Gamma u)(t)\| & \leq\left\|\int_{-\infty}^{t} U(t, \tau) f(\tau, u(\tau)) \mathrm{d} \tau\right\| \\
& \leq N \int_{-\infty}^{t} e^{-\delta(t-\tau)}\|f(\tau, u(\tau))\| \mathrm{d} \tau \\
& \leq \frac{N}{1-e^{-\delta T}} \int_{0}^{T}\|f(\tau, u(\tau))\| \mathrm{d} \tau \\
& \leq \frac{N}{1-e^{-\delta T}} \int_{0}^{T} \Phi_{k_{0}}(\tau) \mathrm{d} \tau \leq k_{0},
\end{aligned}
$$

which implies that $\Gamma u \in \Omega_{k_{0}}$ for every $u \in \Omega_{k_{0}}$.

In the sequel, we show that $\Gamma$ is completely continuous on $\Omega_{k_{0}}$. The proof will be divided into two steps.

Step 1. $\Gamma$ is continuous on $\Omega_{k_{0}}$.

Take $u_{1}, u_{2} \in \Omega_{k_{0}}$. Then it follows from $\left(\mathrm{H}_{3}\right)$ (i) that

$$
\begin{aligned}
& \left\|\left(\Gamma u_{1}\right)(t)-\left(\Gamma u_{2}\right)(t)\right\| \\
& \quad \leq\left\|\int_{-\infty}^{t} U(t, \tau)\left(f\left(\tau, u_{1}(\tau)\right)-f\left(\tau, u_{2}(\tau)\right)\right) \mathrm{d} \tau\right\| \\
& \quad \leq N \int_{-\infty}^{t} e^{-\delta(\mathrm{t}-\tau)}\left\|f\left(\tau, u_{1}(\tau)\right)-f\left(\tau, u_{2}(\tau)\right)\right\| \mathrm{d} \tau \\
& \quad \leq \frac{N}{1-e^{-\delta T}} \int_{0}^{T}\left\|f\left(\tau, u_{1}(\tau)\right)-f\left(\tau, u_{2}(\tau)\right)\right\| \mathrm{d} \tau .
\end{aligned}
$$

This, together with the Lebesgue dominated convergence theorem and the continuity of $f$ with respect to second variable, shows that

$$
\begin{array}{r}
\left\|\left(\Gamma u_{1}\right)(t)-\left(\Gamma u_{2}\right)(t)\right\| \\
\leq \frac{N}{1-e^{-\delta T}} \int_{0}^{T}\left\|f\left(\tau, u_{1}(\tau)\right)-f\left(\tau, u_{2}(\tau)\right)\right\| \mathrm{d} \tau \longrightarrow 0 \\
\text { as } u_{1} \longrightarrow u_{2},
\end{array}
$$

which implies the continuity of $\Gamma$.

Step 2. $\Gamma$ is a compact operator on $\Omega_{k_{0}}$.

For each $\varepsilon>0$, set

$$
\left(\Gamma_{\varepsilon} u\right)(t)=\int_{-\infty}^{t-\varepsilon} U(t, \tau) f(\tau, u(\tau)) \mathrm{d} \tau, \quad u \in \Omega_{k_{0}} .
$$

From $\left(\mathrm{H}_{4}\right)$ it follows that, for each $t \in \mathbb{R}$ and $\varepsilon>0$, the set

$$
\begin{aligned}
& \left\{\int_{-\infty}^{t-\varepsilon} U(t, \tau) f(\tau, u(\tau)) \mathrm{d} \tau ; u \in \Omega_{k_{0}}\right\} \\
& \quad=\left\{U(t, t-\varepsilon) \int_{-\infty}^{t-\varepsilon} U(t-\varepsilon, \tau) f(\tau, u(\tau)) \mathrm{d} \tau ; u \in \Omega_{k_{0}}\right\}
\end{aligned}
$$

is relatively compact in $X$. Thus, for each $t \in \mathbb{R}$ and $\varepsilon>0$, the set $\left\{\left(\Gamma_{\varepsilon} u\right)(t) ; u \in \Omega_{k_{0}}\right\}$ is also relatively compact in $X$. Then, for every $u \in \Omega_{k_{0}}$ and $t \in \mathbb{R}$, as

$$
\begin{aligned}
& \left\|(\Gamma u)(t)-\left(\Gamma_{\varepsilon} u\right)(t)\right\| \\
& \quad \leq\left\|\int_{t-\varepsilon}^{t} U(t, \tau) f(\tau, u(\tau)) \mathrm{d} \tau\right\| \longrightarrow 0 \quad \text { as } \varepsilon \longrightarrow 0
\end{aligned}
$$

in $X$, we conclude, in view of the total boundedness, that for each $t \in \mathbb{R}$, the set $\left\{(\Gamma u)(t) ; u \in \Omega_{k_{0}}\right\}$ is relatively compact in $X$.

Next, we will show that $\left\{\Gamma u ; u \in \Omega_{k_{0}}\right\} \subset P_{T A}(\mathbb{R} ; X)$ is equicontinuous. Taking $t, s \in \mathbb{R}$ with $t>s$, we have

$$
\begin{aligned}
& (\Gamma u)(t)-(\Gamma u)(s) \\
& =\int_{s}^{t} U(t, \tau) f(\tau, u(\tau)) \mathrm{d} s \\
& \quad+\int_{s-\kappa}^{s}(U(t, \tau)-U(s, \tau)) f(\tau, u(\tau)) \mathrm{d} \tau \\
& \quad+\int_{t-M}^{s-\kappa}(U(t, \tau)-U(s, \tau)) f(\tau, u(\tau)) \mathrm{d} \tau \\
& \quad+\int_{-\infty}^{t-M}(U(t, \tau)-U(s, \tau)) f(\tau, u(\tau)) \mathrm{d} \tau \\
& =J_{1}+J_{2}+J_{3}+J_{4},
\end{aligned}
$$

where $\kappa, M$ are positive constants yet to be determined.

Given $\epsilon>0$. We first note that there exist $\eta_{1}>\kappa, \kappa>0$ small enough such that

$$
\begin{aligned}
& \left\|J_{1}\right\| \leq \int_{s}^{t} \Phi_{k_{0}}(\tau) \mathrm{d} \tau \leq \frac{\epsilon}{4} \quad \text { whenever } t-s \leq \eta_{1}, \\
& \left\|J_{2}\right\| \leq 2 N \int_{s-\kappa}^{s} \Phi_{k_{0}}(\tau) \mathrm{d} \tau \leq \frac{\epsilon}{4} .
\end{aligned}
$$

For $J_{4}$, one can take a $M>\left(\eta_{1}+\kappa\right)$ big enough which is independent of $t$ and $s$ such that

$$
\begin{aligned}
\left\|J_{4}\right\| & \leq \int_{-\infty}^{t-M}\|(U(t, \tau)-U(s, \tau))\|_{\mathscr{L}(X)}\|f(\tau, u(\tau))\| \mathrm{d} \tau \\
& \leq N \int_{-\infty}^{t-M}\left(e^{-\delta(t-\tau)}+e^{-\delta(s-\tau)}\right) \Phi_{k_{0}}(\tau) \mathrm{d} \tau \\
& \leq \frac{N\left(1+e^{\delta \eta_{1}}\right) e^{-\delta M}}{1-e^{-\delta T}} \int_{0}^{T} \Phi_{k_{0}}(\tau) \mathrm{d} \tau \leq \frac{\epsilon}{4} .
\end{aligned}
$$

For such fixed $\kappa, M$, it is easy to find that there exists a $d$ big enough such that $|M-\kappa| \leq d T$, which, together with $\left(\mathrm{H}_{3}\right)$ (ii) and (c), yields that

$$
\int_{t-M}^{s-\kappa}\|U(s-\kappa, \tau)\|_{\mathscr{L}(X)}\|f(\tau, u(\tau))\| \mathrm{d} \tau \leq \mathrm{d} N \int_{0}^{T} \Phi_{k_{0}}(\tau) \mathrm{d} \tau .
$$


Therefore, from the continuity of $U(t, s)$ for $t>s$ in the uniform operator topology it follows that

$$
\begin{aligned}
\left\|J_{3}\right\| \leq \int_{t-M}^{s-\kappa}\|(U(t, \tau)-U(s, \tau))\|_{\mathscr{L}(X)}\|f(\tau, u(\tau))\| \mathrm{d} \tau \\
\leq \int_{t-M}^{s-\kappa}\|(U(t, s-\kappa)-U(s, s-\kappa))\|_{\mathscr{L}(X)} \\
\quad \times\|U(s-\kappa, \tau)\|_{\mathscr{L}(X)}\|f(\tau, u(\tau))\| \mathrm{d} \tau \\
\leq d N\|(U(t, s-\kappa)-U(s, s-\kappa))\|_{\mathscr{L}(X)} \int_{0}^{T} \Phi_{k_{0}}(\tau) \mathrm{d} \tau \\
\leq \frac{\epsilon}{4}
\end{aligned}
$$

whenever $t-s \leq \eta_{2}$, where $\eta_{2}$ is small enough.

Thus, from the arguments above one can deduce that there exist $\eta=\min \left\{\eta_{1}, \eta_{2}\right\}$ such that

$$
\|(\Gamma u)(t)-(\Gamma u)(s)\| \leq \epsilon
$$

whenever $t-s \leq \eta$ and $u \in \Omega_{k_{0}}$, which implies that the set $\left\{\Gamma u ; u \in \Omega_{k_{0}}\right\}$ is equicontinuous. Consequently, $\Gamma$ is compact operator on $\Omega_{k_{0}}$ due to Lemma 1.

Now, applying Schauder's fixed point theorem, we deduce that $\Gamma$ has at least one fixed point $u \in P_{T A}(\mathbb{R}, X)$. Moreover, following from the same idea as the last part of the proof in Theorem 8 , we obtain that $u$ is a $T$-antiperiodic mild solution of the problem (2)-(3). This completes the proof.

Remark 10. Theorems 8 and 9 generalize corresponding results for antiperiodic problems due to [12]. Note in particular that Theorems 8 and 9 cover results in [12].

Remark 11. (i) It can be easily shown that if $u$ is antiperiodic with period $T$, then it is periodic with period $2 T$. Hence, from the arguments of Theorems 8 and 9 we can also obtain the existence results of $2 T$-periodic solutions of the problem (2)(3).

(ii) It is clear that if $u$ is periodic with period $2 T, u$ may or may not be antiperiodic with period $T$.

Additional information is contained in the following. We consider the following nonautonomous semilinear parabolic evolution equation with periodic condition

$$
\begin{gathered}
u^{\prime}(t)=A(t) u(t)+f(t, u(t)), \quad t \in \mathbb{R}, \\
u(t+T)=u(t), \quad t \in \mathbb{R} .
\end{gathered}
$$

From the arguments of Theorems 8 and 9 it is easy to see that if

(1) the hypotheses in Theorem 8 are satisfied except that the antiperiodic on $f$ is replaced by the following

$$
f(t+T, u)=f(t, u) \quad \forall t \in \mathbb{R}, u \in X,
$$

then there exists a unique $T$-periodic mild solution for the problem (48);
(2) the hypotheses in Theorem 9 are satisfied except that the antiperiodic conditions on $f$ are replaced by (49), then there exists at least a $T$-periodic mild solution for the problem (48).

The following remark indicates one motivation of the present paper.

Remark 12. As in [25], under certain conditions, the existence result is valid for the case of antiperiodic solutions, while there is no such a result in the periodic case. It is also noted that in dealing with the existence of certain problems, there is an essential difference between the periodic solutions and antiperiodic solutions (see also [26] for more details).

\section{Neutral Problems}

In this section, it is assumed that $(A(t))_{t \in \mathbb{R}}$ has a constant domain $D$ and verifies the conditions of Acquistapace and Terreni $\left(\mathrm{AT}_{1}\right)$ and $\left(\mathrm{AT}_{2}\right)$ with $\lambda_{0}=0$.

If the hypothesis $\left(\mathrm{H}_{1}\right)$ is satisfied, then it follows readily that $t \mapsto A(t)$ is periodic. Also, from Remark 3 it is easy to see that there exist constants $K_{2}>0,0<\mu \leq 1$ such that

$$
\left\|A(t) A^{-1}(0)\right\|_{\mathscr{L}(X)} \leq 1+K_{2}|t|^{\mu}
$$

for all $t \in \mathbb{R}$. Therefore, we deduce that

$$
\left\|A(t) A^{-1}(0)\right\|_{\mathscr{L}(X)} \leq 1+K_{2} T^{\mu}
$$

for all $t \in \mathbb{R}$.

Let $X^{1}$ denote the Banach space $D$ endowed with the graph norm $\|u\|_{1}=\|A(0) u\|$ for $u \in X^{1}$. By $P_{T A}\left(\mathbb{R} ; X^{1}\right)$, we denote the set of all $T$-antiperiodic functions from $\mathbb{R}$ to $X^{1}$. It is clear that $P_{T A}\left(\mathbb{R} ; X^{1}\right)$, equipped with the sup norm, is a Banach space.

In this section, we extend the result obtained in Section 3 to the antiperiodic problem of neutral type (4).

Definition 13. A mild solution to (4) is a function $u \in$ $C_{b}(\mathbb{R} ; X)$ satisfying the integral equation

$$
\begin{aligned}
u(t)= & U(t, s)[u(s)-F(s, u(s))]+F(t, u(t)) \\
& +\int_{s}^{t} U(t, \tau) A(\tau) F(\tau, u(\tau)) \mathrm{d} \tau \\
& +\int_{s}^{t} U(t, \tau) f(\tau, u(\tau)) \mathrm{d} \tau
\end{aligned}
$$

for all $t>s$ and $s \in \mathbb{R}$.

Remark 14. It will be seen later that the last two terms on right side in (52), being integrals in sense of Bocher (see [27]), are reasonable.

To prove the existence of antiperiodic mild solutions to the problem (4), let us introduce the following assumptions:

$\left(\mathrm{H}_{5}\right)$ the function $F: \mathbb{R} \times X \rightarrow X^{1}$ is continuous and $F(t+T,-u)=-F(t, u)$ for all $t \in \mathbb{R}, u \in X$. Moreover, 
(i) there exists a constant $L_{F}$ such that

$$
\|F(t, u)-F(t, v)\|_{1} \leq L_{F}\|u-v\| ;
$$

for all $t \in \mathbb{R}, u, v \in X$;

(ii) there exists a nondecreasing function $\Psi: \mathbb{R}^{+} \rightarrow$ $\mathbb{R}^{+}$such that

$$
\|F(t, u)\|_{1} \leq \Psi(\|u\|) ;
$$

for all $t \in \mathbb{R}, u \in X$, and

$$
\liminf _{r \rightarrow+\infty} \frac{\Psi(r)}{r}=\sigma_{2} \text {. }
$$

$\left(\mathrm{H}_{6}\right)$ Consider $L_{F}\left\|A^{-1}(0)\right\|_{\mathscr{L}(X)}+\left(2 N\left(1+K_{2} T^{\mu}\right) L_{F}+\right.$ $\left.2 N L_{f}\right) / \delta<1$.

$\left(\mathrm{H}_{7}\right)\left(\left\|A^{-1}(0)\right\|_{\mathscr{L}(X)}+\left(N\left(1+K_{2} T^{\mu}\right)\right) / \delta\right) \mu^{\prime}+N \sigma_{1} /\left(1-e^{-\delta T}\right)<$ 1 , where $\mu^{\prime}:=\max \left\{\sigma_{2}, L_{F}\right\}$.

Theorem 15. Under the hypotheses $\left(H_{1}\right),\left(H_{2}\right),\left(H_{5}\right)(i)$, and $\left(\mathrm{H}_{6}\right)$, the problem (4) has a unique T-antiperiodic mild solution.

Proof. From $\left(\mathrm{H}_{5}\right)(\mathrm{i})$ note that $F(\cdot, u(\cdot)) \in P_{T A}\left(\mathbb{R} ; X^{1}\right)$ for each $u \in P_{T A}(\mathbb{R} ; X)$. Define, for $u \in P_{T A}(\mathbb{R} ; X)$,

$$
\begin{aligned}
&(\widetilde{\Gamma} u)(t):= F(t, u(t)) \\
&+\int_{-\infty}^{t} U(t, \tau) \mathscr{P}(\tau) A(\tau) F(\tau, u(\tau)) \mathrm{d} \tau \\
&-\int_{t}^{+\infty} U_{\mathscr{Q}}(t, \tau) \mathcal{Q}(\tau) A(\tau) F(\tau, u(\tau)) \mathrm{d} \tau \\
& t \in \mathbb{R} .
\end{aligned}
$$

Then by (c) and (51) a direct calculation gives

$$
\begin{aligned}
\|(\widetilde{\Gamma} u)(t)\| \leq & \|F(t, u(t))\| \\
& +\int_{-\infty}^{t}\|U(t, \tau) \mathscr{P}(\tau) A(\tau) F(\tau, u(\tau))\| \mathrm{d} \tau \\
& +\int_{t}^{+\infty}\left\|U_{\mathscr{Q}}(t, \tau) \mathcal{Q}(\tau) A(\tau) F(\tau, u(\tau))\right\| \mathrm{d} \tau \\
\leq & \left\|A^{-1}(0)\right\|_{\mathscr{L}(X)}\|F(t, u(t))\|_{1} \\
& +N \int_{-\infty}^{t} e^{-\delta(t-\tau)}\left\|A(\tau) A^{-1}(0)\right\|_{\mathscr{L}(X)} \\
& +N \int_{t}^{+\infty} e^{\delta(t-\tau)}\left\|A(\tau) A^{-1}(0)\right\|_{\mathscr{L}(X)} \\
& \times\|F(\tau, u(\tau))\|_{1} \mathrm{~d} \tau \\
\leq & \left(\left\|A^{-1}(0)\right\|_{\mathscr{L}(X)}+\frac{2 N\left(1+K_{2} T^{\mu}\right)}{\delta}\right) \\
& \times\|F(\cdot, u(\cdot))\|_{P_{T A}\left(\mathbb{R} ; X^{1}\right) \cdot}
\end{aligned}
$$

This proves that $(\widetilde{\Gamma} u)(t)$ is well defined for $t \in \mathbb{R}$ and $\widetilde{\Gamma} u$ is bounded. Moreover, noticing $\left(\mathrm{H}_{5}\right)(\mathrm{i})$ and using a similar argument with that in Lemma 7 one can show easily that $\widetilde{\Gamma}$ maps $P_{T A}(\mathbb{R} ; X)$ into itself.

Let us assume that the mapping $\Gamma$ is defined the same as in Theorem 8. Then from the proof of Theorem 8 with $\left(\mathrm{H}_{2}\right)$ it follows that $\Gamma$ is well defined and maps $P_{T A}(\mathbb{R} ; X)$ into itself.

Now, consider the mapping $\Gamma+\widetilde{\Gamma}$. We see, from the arguments above, that $\Gamma+\widetilde{\Gamma}$ maps $P_{T A}(\mathbb{R} ; X)$ into itself. Also, for $u, v \in P_{T A}(\mathbb{R} ; X), t \in \mathbb{R}$, as

$$
\begin{aligned}
\|(\Gamma u+\widetilde{\Gamma} u)(t)-(\Gamma v+\widetilde{\Gamma} v)(t)\| \\
\leq L_{F}\left\|A^{-1}(0)\right\|_{\mathscr{L}(X)}\|u(t)-v(t)\| \\
\quad+\frac{2 N\left(1+K_{2} T^{\mu}\right) L_{F}+2 N L_{f}}{\delta}\|u-v\|_{P_{T A}(\mathbb{R} ; X)} \\
\leq\left(L_{F}\left\|A^{-1}(0)\right\|_{\mathscr{L}(X)}+\frac{2 N\left(1+K_{2} T^{\mu}\right) L_{F}+2 N L_{f}}{\delta}\right) \\
\quad \times\|u-v\|_{P_{T A}(\mathbb{R} ; X)}
\end{aligned}
$$

in view of $\left(\mathrm{H}_{2}\right)$ and $\left(\mathrm{H}_{5}\right)(\mathrm{i})$, we conclude that $\Gamma+\widetilde{\Gamma}$ is a strict contraction on $P_{T A}(\mathbb{R} ; X)$ due to $\left(\mathrm{H}_{6}\right)$. This allows us to obtain, in view of the contraction mapping principle, that $\Gamma+\widetilde{\Gamma}$ has a unique fixed point $P_{T A}(\mathbb{R}, X)$. Moreover, an application of the same idea as the last part of the proof in Theorem 8 justifies that $u \in P_{T A}(\mathbb{R}, X)$ is a mild solution of (4) if and only if it is a fixed point of $\Gamma+\widetilde{\Gamma}$. The proof is then completed.

The following fixed point theorem plays a key role in the proof of our subsequent result; see, for example, [28].

Lemma 16. Krasnoselskii's fixed point theorem: let $E$ be a Banach space and $B$ be a nonempty closed convex subset of $E$, and let $F_{1}, F_{2}$ be maps of $B$ into $E$ such that $F_{1} x+F_{2} y \in B$ for every pair $x, y \in B$. If $F_{1}$ is a strict contraction and $F_{2}$ is completely continuous, then the equation $F_{1} x+F_{2} x=x$ has a solution on $B$.

Theorem 17. Under the hypotheses $\left(H_{1}\right),\left(H_{7}\right)$, and $\left(H_{3}\right)-\left(H_{5}\right)$ with $\mathscr{P}(t)=I$ for $t \in \mathbb{R}$, the problem (4) has at least one $T$ antiperiodic mild solution.

Proof. From our hypotheses on $F, f$ and $\left(\mathrm{H}_{7}\right)$, it is easy to see that there exists a $k_{0}>0$ such that

$$
\begin{gathered}
\left(\left\|A^{-1}(0)\right\|_{\mathscr{L}(X)}+\frac{N\left(1+K_{2} T^{\mu}\right)}{\delta}\right) \Psi\left(k_{0}\right) \\
+\frac{N}{1-e^{-\delta T}} \int_{0}^{T} \Phi_{k_{0}}(\tau) \mathrm{d} \tau \leq k_{0} .
\end{gathered}
$$


Assume that the mapping $\Gamma$ is defined as in Theorem 9. Let us define the mapping $\widetilde{\Gamma}$ by

$$
\begin{array}{r}
(\widetilde{\Gamma} u)(t):=F(t, u(t))+\int_{-\infty}^{t} U(t, \tau) A(\tau) F(\tau, u(\tau)) \mathrm{d} \tau \\
u \in P_{T A}(\mathbb{R} ; X) .
\end{array}
$$

Note, thanks to the proofs of Theorem 9 with assumptions $\left(\mathrm{H}_{1}\right)$ and $\left(\mathrm{H}_{3}\right)(\mathrm{i})$ and Theorem 15 with assumption $\left(\mathrm{H}_{5}\right)(\mathrm{i})$, that $\Gamma$ and $\widetilde{\Gamma}$ are well defined and map $P_{T A}(\mathbb{R} ; X)$ into itself. Moreover, for every pair $u, v \in \Omega_{k_{0}}$ and $t \in \mathbb{R}$, a direct calculation yields

$$
\begin{aligned}
& \|(\Gamma u)(t)+(\widetilde{\Gamma} v)(t)\| \\
& \leq\left\|A^{-1}(0)\right\|_{\mathscr{L}(X)}\|F(t, u(t))\|_{1} \\
& \quad+N \int_{-\infty}^{t} e^{-\delta(t-\tau)}\left\|A(\tau) A^{-1}(0)\right\|_{\mathscr{L}(X)} \\
& \quad \times\|F(\tau, u(\tau))\|_{1} \mathrm{~d} \tau \\
& \quad+N \int_{-\infty}^{t} e^{-\delta(t-\tau)}\|f(\tau, u(\tau))\| \mathrm{d} \tau \\
& \leq\left(\left\|A^{-1}(0)\right\|_{\mathscr{L}(X)}+\frac{N\left(1+K_{2} T^{\mu}\right)}{\delta}\right) \Psi\left(k_{0}\right) \\
& \quad+\frac{N}{1-e^{-\delta T}} \int_{0}^{T} \Phi_{k_{0}}(\tau) \mathrm{d} \tau \leq k_{0},
\end{aligned}
$$

in view of (59), which implies that $\Gamma u+\widetilde{\Gamma} v \in \Omega_{k_{0}}$ for every pair $u, v \in \Omega_{k_{0}}$.

To obtain the fixed points of $\Gamma+\widetilde{\Gamma}$, we will use Krasnoselskii's fixed point theorem. In what follows, we show that $\Gamma$ and $\widetilde{\Gamma}$ satisfy the conditions of Lemma 16. For $u, v \in \Omega_{k_{0}}$, from $\left(\mathrm{H}_{5}\right)(\mathrm{i})$ we infer that

$$
\begin{aligned}
& \|(\widetilde{\Gamma} u)(t)-(\widetilde{\Gamma} v)(t)\| \\
& \leq L_{F}\left\|A^{-1}(0)\right\|_{\mathscr{L}(X)}\|u(t)-v(t)\| \\
& \quad+\frac{N\left(1+K_{2} T^{\mu}\right) L_{F}}{\delta}\|u-v\|_{P_{T A}(\mathbb{R} ; X)} \\
& \leq\left(L_{F}\left\|A^{-1}(0)\right\|_{\mathscr{L}(X)}+\frac{N\left(1+K_{2} T^{\mu}\right) L_{F}}{\delta}\right) \\
& \quad \times\|u-v\|_{P_{T A}(\mathbb{R} ; X)}
\end{aligned}
$$

for all $t \in \mathbb{R}$, which together with $\left(\mathrm{H}_{7}\right)$ yields that $\widetilde{\Gamma}$ is a contraction on $\Omega_{k_{0}}$. On the other hand, by a similar proof with that in Theorem 9 the mapping $\Gamma$ is completely continuous on $\Omega_{k_{0}}$. Hence, applying Lemma 16 we deduce that $\Gamma+\widetilde{\Gamma}$ has at least one fixed point $u \in \Omega_{k_{0}}$, which is a $T$ antiperiodic mild solution to (4) due to the same idea as the last part of the proof in Theorem 8 . This completes the proof of theorem.
Remark 18. Let us note that in Theorems 8 and 15, exponential dichotomy on evolution equations $U$ is involved. However, as can be seen from the proofs of Theorems 9 and 17, such condition is not enough to obtain our desired results and therefore is replaced by the special one: $U$ is exponentially stable.

\section{Application}

In this section, we give an example to illustrate our abstract results, which do not aim at generality but indicate how our theorems can be applied to concrete problem.

Consider the antiperiodic problem for partial differential equation in the form

$$
\begin{array}{r}
\frac{\partial}{\partial t} u(t, x)=a(t) \frac{\partial^{2} u(t, x)}{\partial x^{2}}+g(t, x, u(t, x)), \\
t \in \mathbb{R}, \quad x \in[0, \pi], \\
u(t+T, x)=-u(t, x), \quad t \in \mathbb{R}, x \in[0, \pi],
\end{array}
$$

supplemented with homogeneous Dirichlet boundary condition $u(t, 0)=u(t, \pi)=0(t \in \mathbb{R})$, where $g: \mathbb{R} \times[0, \pi] \times \mathbb{R} \rightarrow$ $\mathbb{R}, a: \mathbb{R} \rightarrow \mathbb{R}$ are given functions which will be specified later.

Here, our objective is to show the existence of $T$ antiperiodic solutions for the antiperiodic problem (63).

Take $X=\left(L^{2}(0, \pi) ;(\cdot, \cdot)_{2}\right)$ and define the operator $B$ : $D(B) \subset X \rightarrow X$ by $B u=\partial^{2} u / \partial x^{2}, u \in D(B)$, where

$$
D(B)=H^{2}(0, \pi) \cap H_{0}^{1}(0, \pi) .
$$

It is well-known that $B$ has a discrete spectrum and its eigenvalues are $-n^{2}, n \in \mathbb{N}^{+}$with the corresponding normalized eigenvectors $y_{n}(x)=\sqrt{(2 / \pi)} \sin (n x)$.

Assume that $a$ is a Hölder continuous function such that

$$
a(t+T)=a(t) \quad \forall t \in \mathbb{R}, \inf _{t \in \mathbb{R}} a(t) \geq c>0 .
$$

Take $g(t, x, u(t, x))=b(t) \sin u(t, x)$, where

$$
b(t+T)=b(t) \quad \text { for a.e. } t \in \mathbb{R},\left.b\right|_{[0, T]} \in L\left(0, T ; \mathbb{R}^{+}\right) .
$$

Define

$$
\begin{gathered}
D(A(t))=D(B), \quad t \in \mathbb{R}, \\
A(t) u=a(t) B u, \quad u \in D(A(t)), \\
u(t)(x)=u(t, x), \\
f(t, u(t))(x)=g(t, x, u(t, x)) .
\end{gathered}
$$

It follows from [19, Lemma 6.1 in Chapter 7] that there are constants $\theta \in(\pi / 2, \pi)$ and $K_{1} \geq 0$ such that $A(t)$ satisfy

$$
\begin{gathered}
\Sigma_{\theta}:=\{\lambda \in \mathbb{C} \backslash\{0\} ;|\lambda| \leq \theta\} \cup\{0\} \subset \rho(A(t)), \\
\|R(\lambda, A(t))\| \leq \frac{K_{1}}{1+|\lambda|}
\end{gathered}
$$


for all $\lambda \in \Sigma_{\theta} \cup\{0\}$ and $t \in \mathbb{R}$. Moreover, we note that for $\lambda \in \Sigma_{\theta} \cup\{0\}, t, s \in \mathbb{R}, u \in X$,

$$
\begin{gathered}
R(\lambda, A(t)) u=\sum_{n=1}^{\infty} \frac{1}{\lambda+n^{2} a(t)}\left(u, y_{n}\right) y_{n}, \\
A(t) R(\lambda, A(t))[R(0, A(t))-R(0, A(s))] u \\
=\sum_{n=1}^{\infty} \frac{1}{\lambda+n^{2} a(t)} \frac{a(t)-a(s)}{a(s)}\left(u, y_{n}\right) y_{n}
\end{gathered}
$$

from which we see that for, $\lambda \in \Sigma_{\theta} \cup\{0\}, t, s \in \mathbb{R}$

$$
\begin{aligned}
& \|A(t) R(\lambda, A(t))[R(0, A(t))-R(0, A(s))]\| \\
& \leq \frac{\|R(\lambda, A(t))\| \cdot|a(t)-a(s)|}{a(s)} .
\end{aligned}
$$

Accordingly, $A(t)$ satisfy the conditions $\left(\mathrm{AT}_{1}\right)$ and $\left(\mathrm{AT}_{2}\right)$. Thus, the family $(A(t))_{t \in \mathbb{R}}$ generates an evolution family $U$ :

$$
\begin{array}{r}
U(t, s) u=\sum_{n=1}^{\infty} e^{-n^{2} \int_{s}^{t} a(\tau) \mathrm{d} \tau}\left(u, y_{n}\right) y_{n}, \\
\text { for }-\infty \leq s \leq t<\infty, \quad u \in X .
\end{array}
$$

A direct calculation gives

$$
\|U(t, s)\| \leq e^{-c(t-s)} \quad \text { for }-\infty \leq s \leq t<+\infty .
$$

Also, $U$ is compact due to the boundedness of $A(t) U(t, s)$ for $-\infty \leq s<t<+\infty$ (cf. [19, Theorem 6.1 in Chapter 5]). Moreover, it is easy to verify that $U(t+T, s+T)=U(t, s)$ for all $t \geq s$.

On the other hand, observe that $f: \mathbb{R} \times X \rightarrow X$ is a Carathéodory function, $f(t+T,-u)=-f(t, u)$ for all $t \in \mathbb{R}$, $u \in X$, and

$$
\|f(t, u)\| \leq \sqrt{\pi} b(t) \quad \text { for a.e. } t \in[0, T] \text { and all } u \in X,
$$

which implies that hypothesis $\left(\mathrm{H}_{3}\right)$ is satisfied with

$$
\Phi_{r}(t)=\sqrt{\pi} b(t), \quad \sigma_{1}=0 .
$$

Therefore, the antiperiodic problem (63) can be transformed into the abstract problem (2)-(3) and assumptions $\left(\mathrm{H}_{1}\right),\left(\mathrm{H}_{3}\right)$, and $\left(\mathrm{H}_{4}\right)$ hold with $\mathscr{P}(t)=I$ for $t \in \mathbb{R}$. Hence, (63) has at least one $T$-antiperiodic mild solution due to Theorem 9 .

\section{Conflict of Interests}

The authors declare that there is no conflict of interests regarding the publication of this paper.

\section{Acknowledgments}

This research was supported by the Natural Science Foundations of China (no. 11271309, 11101202), the Specialized
Research Fund for the Doctoral Program of Higher Education (20114301110001), and Hunan Provincial Natural Science Foundation of China (12JJ2001). The authors also want to express their thanks to the anonymous referees for their suggestions and comments that improved the quality of the paper.

\section{References}

[1] M. T. Batchelor, R. J. Baxter, M. J. O’Rourke, and C. M. Yung, "Exact solution and interfacial tension of the six-vertex model with anti-periodic boundary conditions," Journal of Physics A: Mathematical and General, vol. 28, no. 10, pp. 2759-2770, 1995.

[2] L. L. Bonilla and F. J. Higuera, "The onset and end of the Gunn effect in extrinsic semiconductors," SIAM Journal on Applied Mathematics, vol. 55, no. 6, pp. 1625-1649, 1995.

[3] D. S. Kulshreshtha, J. Q. Liang, and H. J. W. Müller-Kirsten, "Fluctuation equations about classical field configurations and supersymmetric quantum mechanics," Annals of Physics, vol. 225, no. 2, pp. 191-211, 1993.

[4] H. Okochi, "On the existence of periodic solutions to nonlinear abstract parabolic equations," Journal of the Mathematical Society of Japan, vol. 40, no. 3, pp. 541-553, 1988.

[5] H. Okochi, "On the existence of anti-periodic solutions to a nonlinear evolution equation associated with odd subdifferential operators," Journal of Functional Analysis, vol. 91, no. 2, pp. 246-258, 1990.

[6] H. Okochi, "On the existence of anti-periodic solutions to nonlinear parabolic equations in noncylindrical domains," Nonlinear Analysis: Theory, Methods \& Applications, vol. 14, no. 9, pp. 771-783, 1990.

[7] A. Haraux, "Anti-periodic solutions of some nonlinear evolution equations," Manuscripta Mathematica, vol. 63, no. 4, pp. 479-505, 1989.

[8] A. R. Aftabizadeh, N. H. Pavel, and Y. K. Huang, "Antiperiodic oscillations of some second-order differential equations and optimal control problems," Journal of Computational and Applied Mathematics, vol. 52, no. 1-3, pp. 3-21, 1994.

[9] S. Aizicovici and N. H. Pavel, "Anti-periodic solutions to a class of nonlinear differential equations in Hilbert space," Journal of Functional Analysis, vol. 99, no. 2, pp. 387-408, 1991.

[10] Z. H. Liu, "Anti-periodic solutions to nonlinear evolution equations," Journal of Functional Analysis, vol. 258, no. 6, pp. 2026-2033, 2010.

[11] Y. Q. Chen, J. J. Nieto, and D. O’Regan, “Anti-periodic solutions for evolution equations associated with maximal monotone mappings," Applied Mathematics Letters, vol. 24, no. 3, pp. 302307, 2011.

[12] Q. Liu, "Existence of anti-periodic mild solutions for semilinear evolution equations," Journal of Mathematical Analysis and Applications, vol. 377, no. 1, pp. 110-120, 2011.

[13] R. N. Wang, Q. M. Xiang, and Y. Zhou, "Asymptotically periodic solutions to nonlocal Cauchy problems governed by compact evolution families," Nonlinear Oscillations, vol. 16, no. 1, pp. 1428, 2013.

[14] Y. Wang, "Antiperiodic solutions for dissipative evolution equations," Mathematical and Computer Modelling, vol. 51, no. 5-6, pp. 715-721, 2010.

[15] R. N. Wang and D. H. Chen, "Anti-periodic problems for semilinear partial neutral evolution equations," Electronic Journal 
of Qualitative Theory of Differential Equations, no. 16, pp. 1-16, 2013.

[16] P. Acquistapace, "Evolution operators and strong solutions of abstract linear parabolic equations," Differential and Integral Equations, vol. 1, no. 4, pp. 433-457, 1988.

[17] P. Acquistapace and B. Terreni, "A unified approach to abstract linear nonautonomous parabolic equations," Rendiconti del Seminario Matematico della Università di Padova, vol. 78, pp. 47-107, 1987.

[18] H. Amann, Linear and Quasilinear Parabolic Problems, vol. 89 of Monographs in Mathematics, Birkhäuser, Berlin, Germany, 1995.

[19] A. Pazy, Semigroups of Linear Operators and Applications to Partial Differential Equations, vol. 44 of Applied Mathematical Sciences, Springer, New York, NY, USA, 1983.

[20] A. Yagi, "Abstract quasilinear evolution equations of parabolic type in Banach spaces," Bollettino dell'Unione Matematica Italiana B, vol. 5, no. 2, pp. 341-368, 1991.

[21] A. Yagi, "Parabolic evolution equations in which the coefficients are the generators of infinitely differentiable semigroups. II," Funkcialaj Ekvacioj, vol. 33, no. 1, pp. 139-150, 1990.

[22] K. J. Engel and R. Nagel, One-Parameter Semigroups for Linear Evolution Equations, vol. 194 of Graduate Texts in Mathematics, Springer, Berlin, Germany, 2000.

[23] D. Henry, Geometric Theory of Semilinear Parabolic Equations, vol. 840 of Lecture Notes in Mathematics, Springer, Berlin, Germany, 1981.

[24] L. Maniar and R. Schnaubelt, Almost Periodicity of Inhomogeneous Parabolic Evolution Equations, vol. 234 of Lecture Notes in Pure and Applied Mathematics, Marcel Dekker, New York, NY, USA, 2003.

[25] Y. Q. Chen, X. D. Wang, and H. X. Xu, "Anti-periodic solutions for semilinear evolution equations," Journal of Mathematical Analysis and Applications, vol. 273, no. 2, pp. 627-636, 2002.

[26] X. Liu and J. Cao, "Exponential stability of anti-periodic solutions for neural networks with multiple discrete and distributed delays," Journal of Systems and Control Engineering, vol. 223, pp. 299-308, 2009.

[27] C. M. Marle, Mesures et Probabilités, Hermann, Paris, France, 1974.

[28] M. A. Krasnosel'skii, Topological Methods in the Theory of Nonlinear Integral Equations, Cambridge University Press, New York, NY, USA, 1964. 


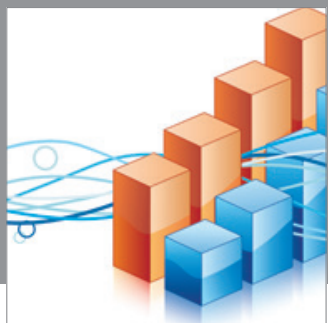

Advances in

Operations Research

mansans

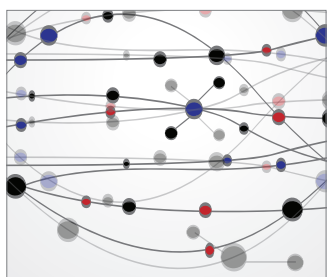

The Scientific World Journal
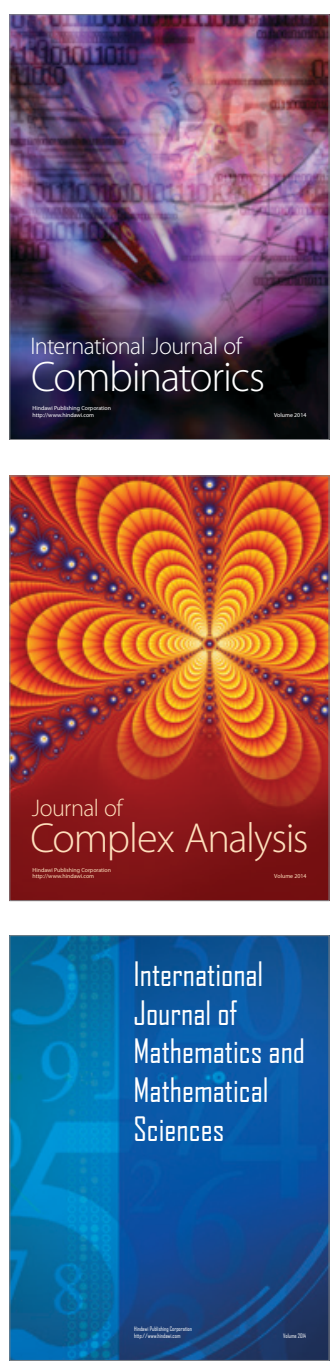
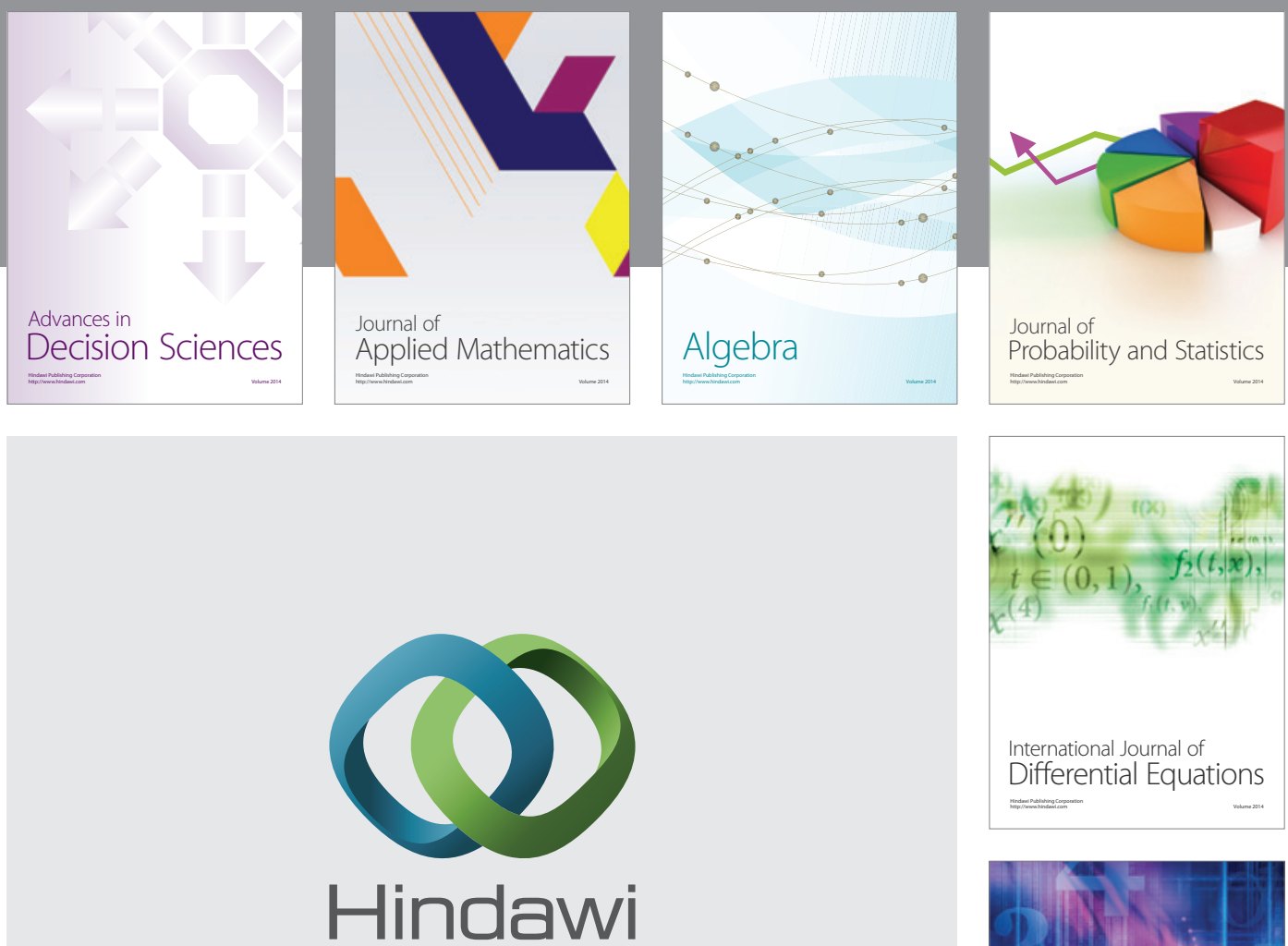

Submit your manuscripts at http://www.hindawi.com
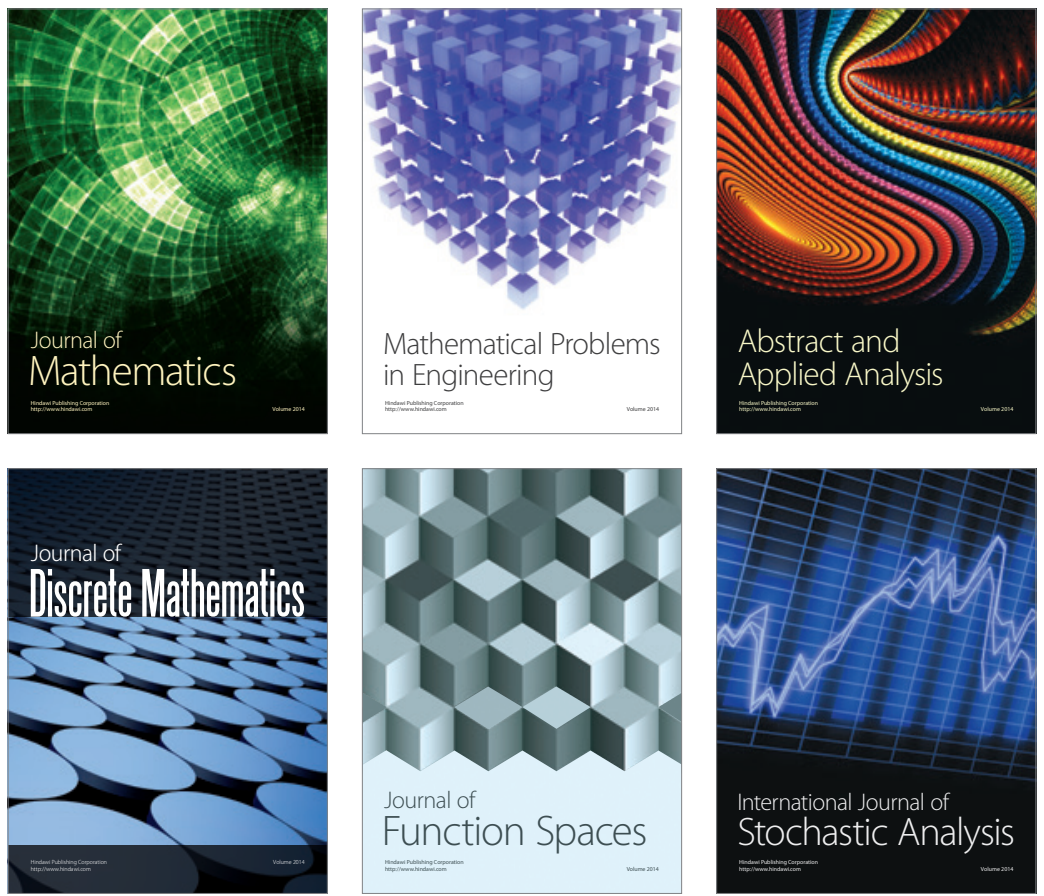

Journal of

Function Spaces

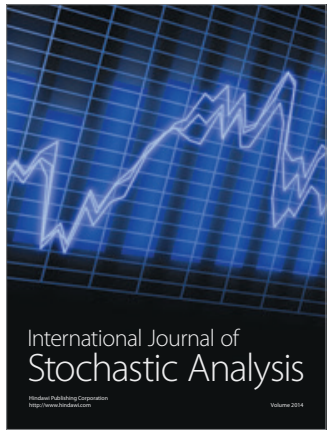

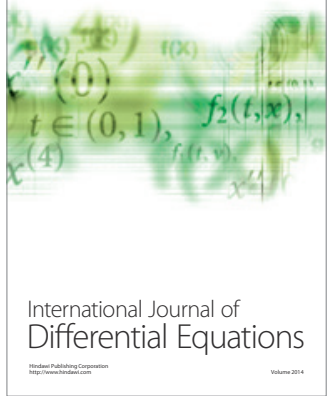
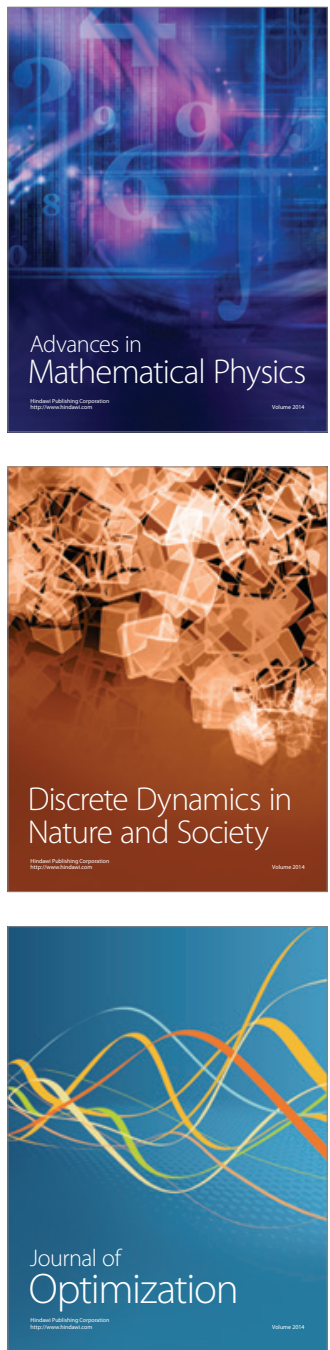